\title{
Analysis on cracking of repaired tubing used in surface gas injection pipeline in a western oilfield in China
}

\author{
Lei $\mathrm{Li}^{1, \mathrm{a}}$, Jing Yuan ${ }^{2}$, Xianren Kuang ${ }^{1}$, Juntao Yuan ${ }^{1}$, Wenhong Liu ${ }^{1}, \mathrm{Kai}_{\mathrm{Lin}}{ }^{1}$ and Han Ding ${ }^{1}$ \\ ${ }^{1}$ CNPC Tubular Goods Research Institute, Xi'an 710077, China \\ ${ }^{2}$ Tarim Oilfield Company of PetroChina, Korla 841000, China
}

\begin{abstract}
According to the cracking accident of repaired tubing used in surface gas injection pipeline in a western oilfield in China, the cause of the failure tubing was analysed by means of macroscopic analysis, non-destructive testing, chemical analysis, mechanical testing, metallographic examination, macro and micro fracture analysis. Combined with the working environment of the repaired tubing, conclusions were made that the failure cause of the tubing was sulfide stress corrosion cracking (SSCC) and the crack originated from the clamp area of make -and-break test in the outside surface of the tubing. Finally, suggestions and measures for prevention congener failure were put forward.
\end{abstract}

Keywords: repaired tubing; gas injection pipeline; Failure analysis; Sulfide stress corrosion cracking.

\section{Introduction}

As tubing is oil industry consumables, a lot of new tubing are purchased annually and also a large number of old tubing are scrapped. If the old tubing is only piled up in the material field, it is bound to cause great waste. At present, the old tubing after evaluation and repair is used again in all oil and gas fields in China. This not only solves the problem of the waste of the old tubing, but also greatly saves the cost of new tubing. Usually for screw wear and bearing capacity meeting the requirements of the old tubing, heavy vehicle thread repairing method can be used, which continues to be used as tubing. Moreover, for the old tubing which can't be repaired, it can be application to surface gathering pipeline after repaired [1]. It's reported that repaired tubing for surface gathering pipeline is not only technically feasible, but also economically reduce the pipeline replacement costs [2].

However, with more and more repaired tubing for surface gathering pipeline, some difficult problems also appear such as pipeline cracking, etc., which result in a greater economic losses to all oil field companies. In this paper, through the comprehensive analysis of the cracking accident of the old tubing used in surface gas injection pipeline in a western oilfield in China, the cause and mechanism of the failure are to find out, and suggestions and reference are provided for the safe use of the old tubing.

${ }^{a}$ Corresponding author : 115457885@163.com 


\section{Experimental}

The failure tubing for investigation was subjected to the following procedures to clear cracking reasons.

\subsection{Visual examination and non-destructive testing}

The inner and outer surface of the tubing was examination visually. The defect and crack in the surface of the tubing were investigated primarily, and the typical macro morphology was taken using the camera (NIKON L100).

Then non-destructive testing of the tubing was conducted via magnetic particle testing and ultrasonic testing, which focused on the defect of the tubing body.

\subsection{Physico-chemical properties testing}

The chemical compositions of the tubing were analysed by a direct reading spectrometer (ARL 4460) based on ASTM A751-14a. The metallographic structure and inclusions were analysed by metallographic microscope (MEF3A) on the basis of GB/T 13298-1991[3], GB/T 10561-2005[4] and GB/T 6394-2002[5].

Longitudinal tensile specimens were taken from the failure tubing. The room temperature tensile properties test was conducted with a specification of $19.1 \mathrm{~mm} \times 50 \mathrm{~mm}$ (width $\times$ gauge) by tensile testing machine (UTM5305), which is on the basis of ASTM A370-15. Impact test specimens (transverse) are machined from the failure tubing. Charpy V-notch impact tests shall be conducted as specified in ASTM E23-12c with a specification of $5 \mathrm{~mm} \times 10 \mathrm{~mm} \times 55 \mathrm{~mm}$ by means of impact testing machine (PIT302D). The through-wall hardness (transverse) testing with a Rockwell C-scale is carried out according to ASTM E 18-15 by hardness tester (RB 2002).

\subsection{Fracture surface observation}

Morphologies and characteristics of the fracture surface of the tubing were investigated by means of a Scanning Electron Microscopy (SEM, Philips xl-30), and chemical compositions of the fracture surface films were tested by Energy Dispersion Spectrum (EDS, INCA).

\section{Results and discussion}

\subsection{Macroscopic analysis and non-destructive testing}

Figure 1 shows macroscopic photos of the failure tubing. The outer wall of the tubing has a length of about $190 \mathrm{~mm}$ of the longitudinal crack extending to the disappearance of the external thread. The internal and external walls of it don't have obvious corrosion (Figure 1a and Figure 1b), while the external wall in the vicinity of the crack has a relatively shallow clamp printing (Figure 1c). Moreover, the deep clamp printing can also be seen in the region to the other parts of the ring (Figure 1d), and the surface of the external thread was no damage and intact appearance.

The failure tubing was subjected to ultrasonic detection and the inside and outside surface of the magnetic particle testing. Results show that the body of the tubing produces a length of about $190 \mathrm{~mm}$ longitudinal penetrating crack extended to the external thread disappear. Magnetic particle testing results are shown in Figure 2. 

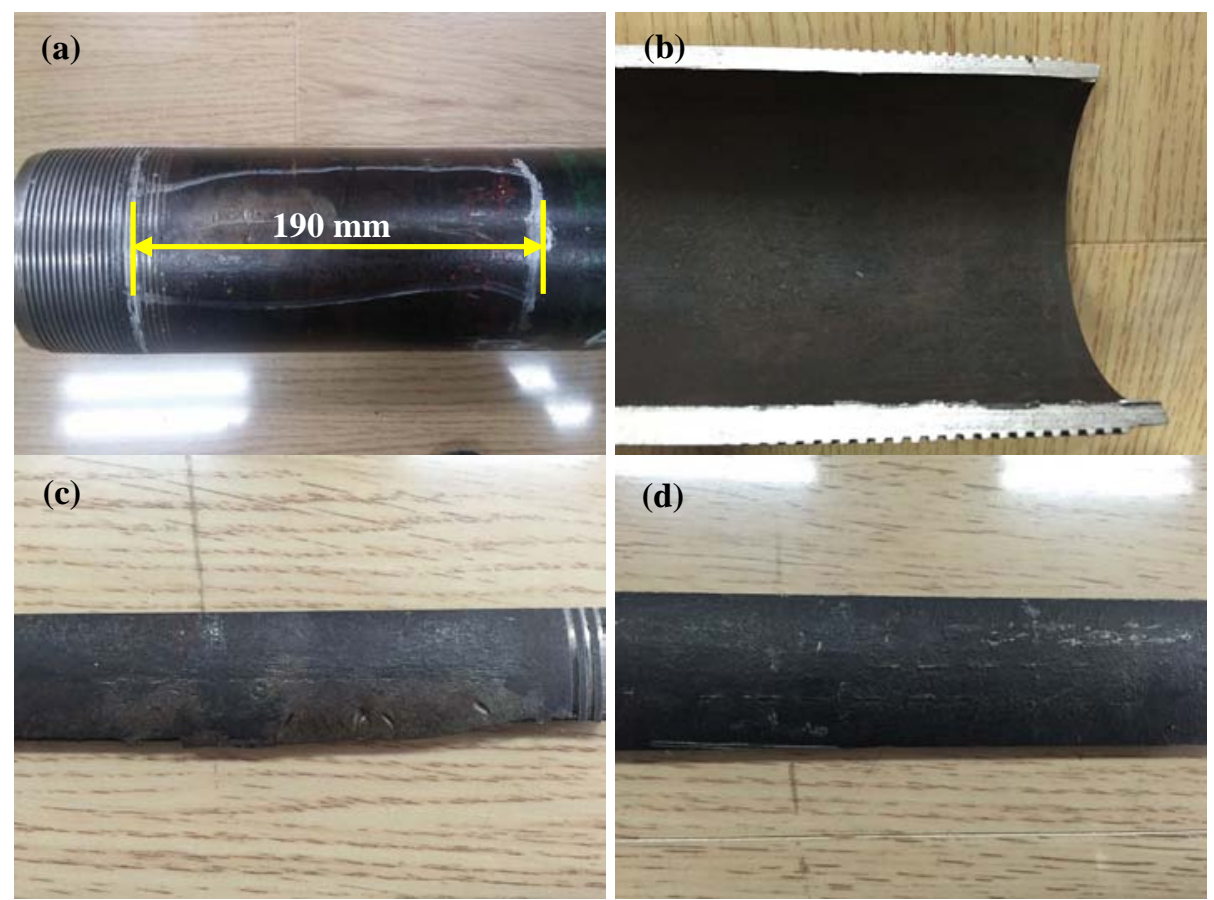

Figure 1. Macroscopic photos of the failure tubing
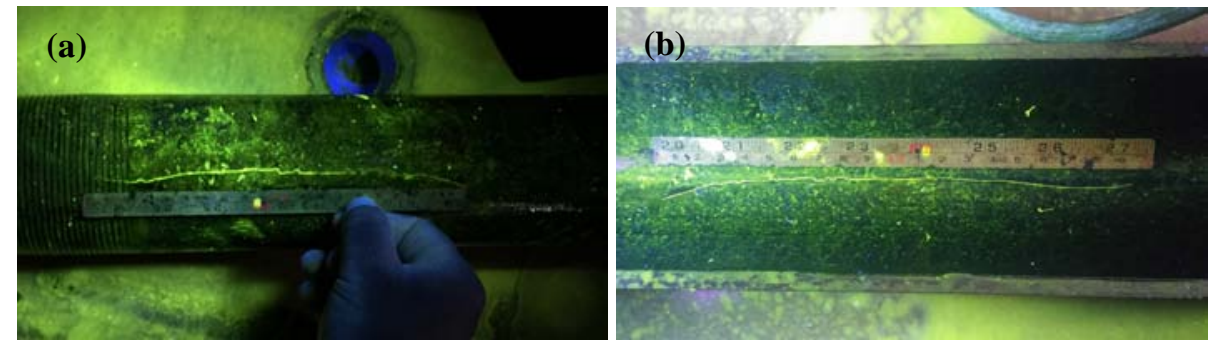

Figure 2. The results of magnetic particle testing of internal and external surface of the failure tubing: (a) external surface and (b) internal surface

\subsection{Fracture macroscopic analysis}

After the crack was opened by mechanical means, the macroscopic fracture with cleared by cellulose acetate was observed by optical microscope as shown in Figure 3. It shows a smooth and blackened fracture with the radial pattern obviously, which converges to the outer wall of the tubing body. Those belong to characteristics of brittle fracture. Figure 4 the longitudinal position of the crack source of the fracture. From Figure 4, we can see that the distance between the source of the fracture surface and the disappearance of the external thread is $40 \mathrm{~mm}$. According to the make-and-break experience and macroscopic observation, it can be found this place is the clamp area of make-and-break test of tubing exactly and clamp marks are remarkable. 


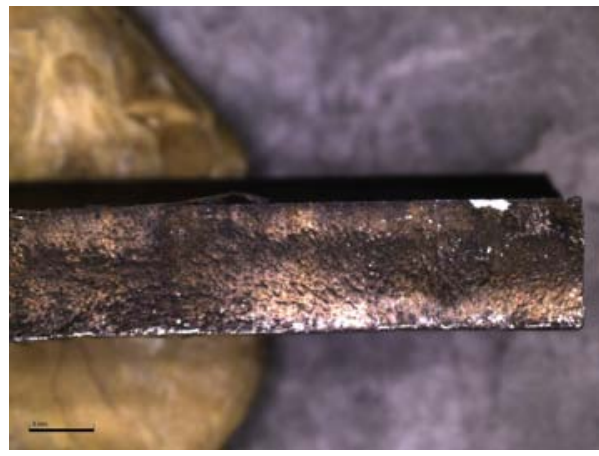

Figure 3. The macro morphology of fracture

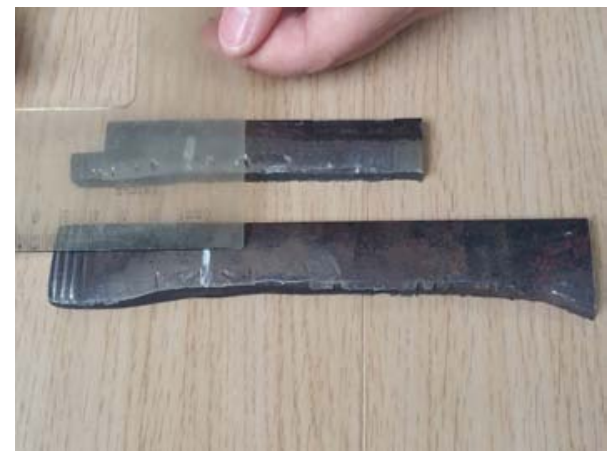

Figure 4. The location of crack source

\subsection{Chemical composition analysis}

Chemical compositions of the failure tubing are shown in Table 1, which are compared with the technical requirements of API SPEC 5CT (Ninth Edition) on P110 steel grade tubing. Results show that all the chemical components meet the requirements of API SPEC 5CT (Ninth Edition).

Table 1. Chemical compositions of the failure tubing (wt. \%)

\begin{tabular}{lllllllllll}
\hline Element & $\mathrm{C}$ & $\mathrm{Si}$ & $\mathrm{Mn}$ & $\mathrm{P}$ & $\mathrm{S}$ & $\mathrm{Cr}$ & $\mathrm{Mo}$ & $\mathrm{Ni}$ & $\mathrm{Ti}$ & $\mathrm{Cu}$ \\
\hline Measured value & 0.25 & 0.24 & 1.42 & 0.020 & 0.0040 & 0.83 & 0.10 & 0.041 & 0.013 & 0.012 \\
\hline API SPEC 5CT & - & - & - & $\leq 0.030$ & $\leq 0.030$ & - & - & - & - & - \\
\hline
\end{tabular}

\subsection{Mechanical property analysis}

Values of the tensile test and the impact test are shown in Table 2 and Table 3 separately, which are compared with requirements of API SPEC 5CT (Ninth Edition). Results show that the tensile and impact properties of the failure tubing are satisfied with the technical requirements of API SPEC 5CT (Ninth Edition) for P110 pipe. In addition, the hardness value (HRC) is shown in Table 4.

Table 2. The results of tensile test

\begin{tabular}{lccc}
\hline & $\begin{array}{c}\text { Tensile strength } \\
(\mathrm{MPa})\end{array}$ & $\begin{array}{c}\text { Yield strength }(0.6 \% \mathrm{EUL}) \\
(\mathrm{MPa})\end{array}$ & $\begin{array}{c}\text { Elongation } \\
(\%)\end{array}$ \\
\hline Measured value & 981967978 & 910907911 & 181819 \\
API SPEC 5CT & $\geq 862$ & $758 \sim 965$ & $\geq 12$ \\
\hline
\end{tabular}

Table 3. The results of Charpy impact test

\begin{tabular}{lccc}
\hline & Temperature $\left({ }^{\circ} \mathrm{C}\right)$ & Absorbed energy $(\mathrm{J})$ & Percent of shearing area $(\%)$ \\
\hline Measured value & 0 & 636768 & 100100100 \\
API SPEC 5CT & $0 \pm 3$ & $\geq 23$ & - \\
\hline
\end{tabular}

Table 4. The hardness value (HRC)

\begin{tabular}{|c|c|c|}
\hline Location & Hardness value & Average value \\
\hline The first quadrant & $\begin{array}{lll}30.3 & 30.7 & 30.6\end{array}$ & 30.5 \\
\hline The second quadrant & $\begin{array}{lll}30.7 & 30.8 & 30.7\end{array}$ & 30.7 \\
\hline The third quadrant & $26.427 .7 \quad 28.1$ & 27.4 \\
\hline The fourth quadrant & 26.527 .128 .6 & 27.4 \\
\hline
\end{tabular}




\subsection{Analysis of metallographic structure}

The samples were cut from the body of the tubing and the vicinity of the crack of the tubing. Results of metallographic observation are as shown in Figure 5 to 7 . It can be seen from the figures that body tissue of the tubing is tempered sorbite, Grain size is 9.0 grade and non-metallic inclusions is A0.5, B0.5 and D0.5 (Figure 5). The tissue near the crack has no abnormalities (Figure 6), the crack of the tubing body is extended from the external surface to the inner surface (Figure 7).
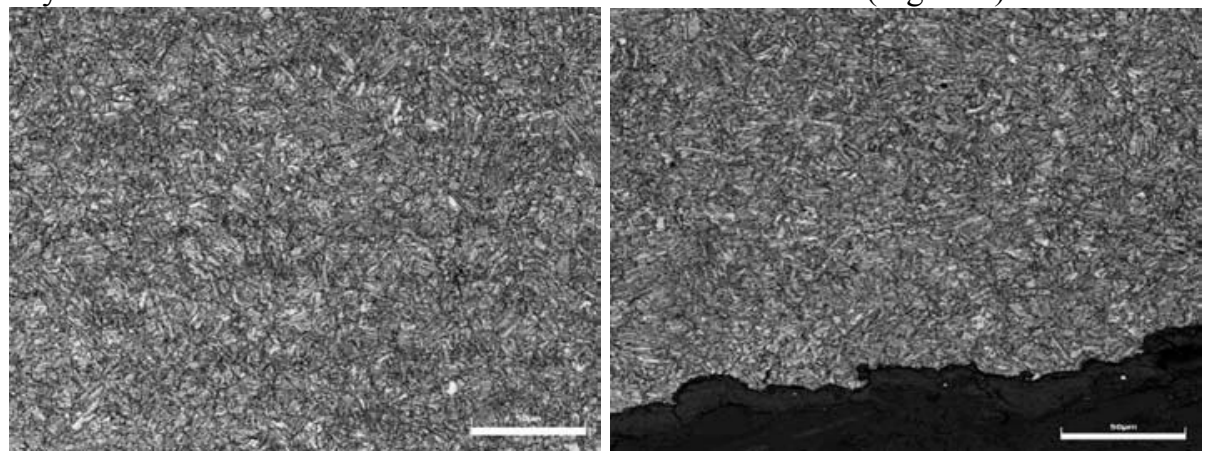

Figure 5. The body tissue of the tubing

Figure 6. The tissue of the tubing near crack

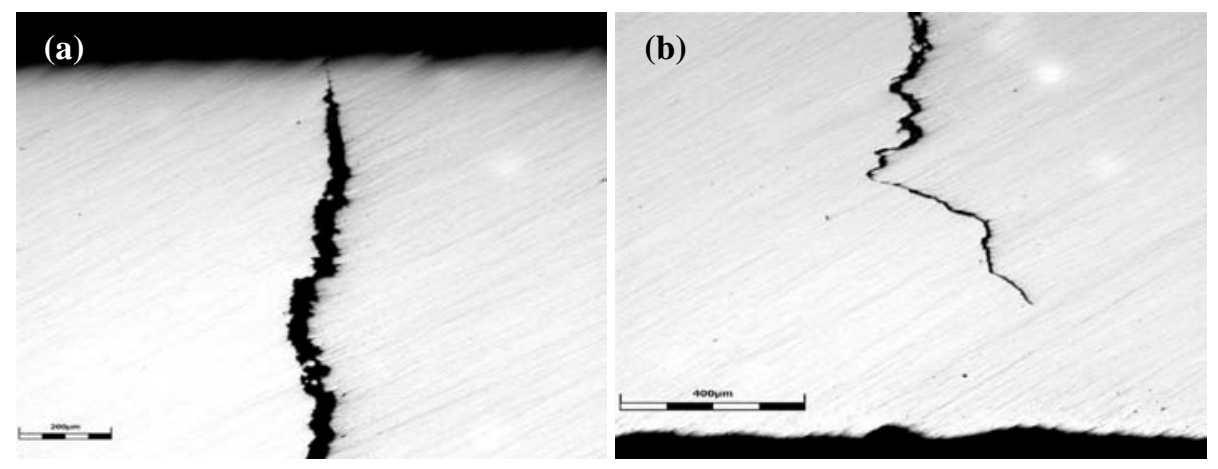

Figure 7. Morphology of the longitudinal crack in the body of the tubing: (a) crack near the outer surface and (b) crack near the inner surface

\subsection{Microscopic analysis of the fracture}

Figure 8 shows microscopic morphology of the fracture. As can be seen from the diagram, the fracture at the side of the outer wall has obvious radial pattern and its convergence is for the crack source. It shows a flat fracture with no obvious plastic deformation (Figure 8a). Intergranular traces exist in the source region and the growth region. Moreover, intergranular secondary cracks are in the crosssection of the fracture (Figure $8 \mathrm{~b}$ and $8 \mathrm{c}$ ). EDS analysis of the fracture surface shows the content of element $\mathrm{S}$ is relatively high, as shown in Table 4. 

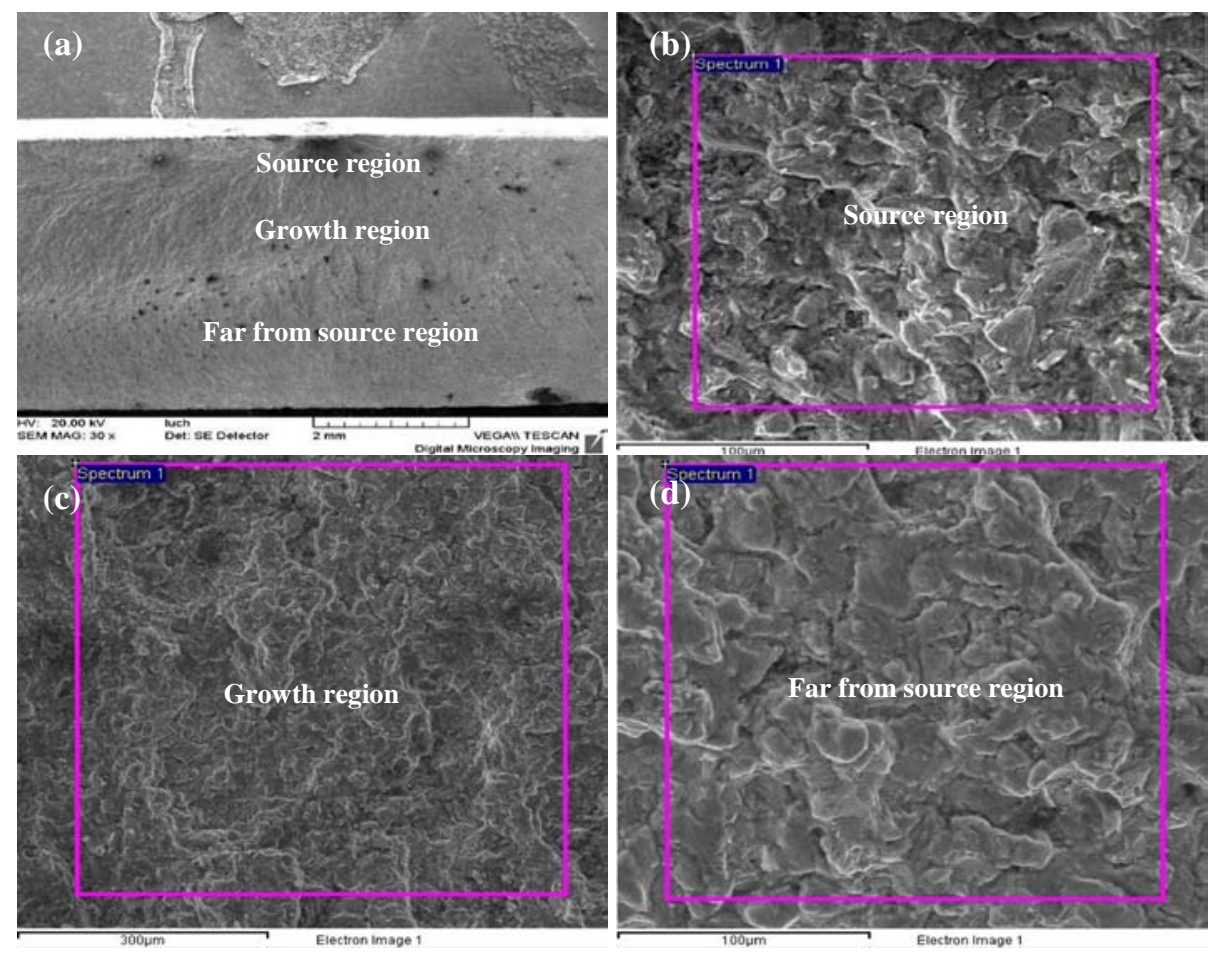

Figure 8. Microscopic morphology of the fracture

Table 5. Results of energy spectrum analysis at different locations of the fracture (wt. \%)

\begin{tabular}{ccccccc}
\hline Element & $\mathrm{C}$ & $\mathrm{O}$ & $\mathrm{S}$ & $\mathrm{Cr}$ & $\mathrm{Mn}$ & $\mathrm{Fe}$ \\
\hline The content of source region & 8.07 & 13.97 & $\mathbf{8 . 1 6}$ & $/$ & 1.29 & 68.51 \\
The content of growth region & 11.63 & 21.05 & $\mathbf{5 . 8 1}$ & 0.81 & 1.28 & 59.41 \\
$\begin{array}{c}\text { The content of the area } \\
\text { far from source region }\end{array}$ & 8.10 & 16.10 & $\mathbf{1 3 . 4 1}$ & 0.92 & $/$ & 61.47 \\
\hline
\end{tabular}

\section{Failure mechanism analysis}

According to the physical and chemical properties of the failure tubing above, it is known that the chemical composition and mechanical properties of the failure tubing are in accordance with the technical requirements of API SPEC 5CT (Ninth Edition) for the P110 pipe, and the metallographic structure is not abnormal.

The observation of the macro morphology of longitudinal cracking of the tubing shows that the fracture is flat with no obvious plastic deformation. Meanwhile, macroscopic fracture with cleared by cellulose acetate is blackened and has the radial pattern obviously, which converges to the outer wall of the tubing body. Those belong to characteristics of brittle fracture, which meet macro fracture characteristics of sulfide stress corrosion cracking (SSCC). The micro morphology analysis of the fracture surface can be known that the characteristic of intergranular traces exists, and intergranular secondary cracks are in the cross-section of the fracture, which is in accordance with the micro fracture characteristic of SSCC [6]. In addition, EDS analysis of the cross-section of the fracture also shows some element $\mathrm{S}$. By the macroscopic and microscopic characteristics of the above section, it can be determined that the failure tubing is a typical SSCC, and the crack originates from the outside surface of the tubing.

The repaired tubing is used in ground pipeline for conveying natural gas. As the external environment of that don't contain the sensitive medium of SSCC, it is impossible that SSCC 
originating from the outside surface of the tubing occurs under normal circumstances. It can be concluded that the tubing has served in the well containing the sensitive medium (eg. hydrogen sulfide) and the external surface of the tubing has been eroded, especially for the surface defect. The residual clamp printings as the outer surface defects of the tubing are more likely to suffer from sensitive medium erosion [7], which results in damage of the outer surface of the tubing in the vicinity of clamp printings. However, the stress is too small to cracking of the tubing. Subsequently when applied for the ground pipeline, the tubing cracks due to the relatively high pressure of the pipe.

Studies [8-9] have shown that metal material or component occurring SSCC must have three conditions: (1) the specific environment with hydrogen sulfide medium; (2) the sensitivity of materials to SSCC; (3) component in the service process to bear a certain load.

First, the repaired tubing has served in well, but its served history is unknown. According to the field research, the tubing may have served in the environment with element $\mathrm{S}$, which agrees with the experimental results. EDS of the fracture also confirms that the cracking surface contains a large number of element S. Secondly, the hardness value of the tubing is about 30 HRC. Research [7,9,10] shows that SSCC of a carbon steel occurs with the lowest hardness value of 22 HRC. So the tubing has a large sensitivity of SSCC. Finally, the service pressure of $9.8 \mathrm{MPa}$ (inner pressure) of the tubing is far less than that of the hydrostatic test $(69 \mathrm{MPa})$ in API Spec 5CT (Ninth Edition). However, pressure bearing capacity of the tubing has reduced greatly because the external surface of it has been eroded by the sensitive medium of SSCC, which leads to SSCC under low stress. In summary, the three conditions of SSCC occurring have been met, so it can be further determined that failure mode of the tubing is SSCC.

\section{Conclusions and suggestions}

(1) The chemical composition and mechanical properties of the repaired tubing are satisfied with the technical requirements of API SPEC 5CT (Ninth Edition) for P110 tubing, and the metallographic structure is not abnormal.

(2) The failure cause of the tubing is SSCC and the crack originates from the clamp area of make and-break test in the outside surface of the tubing.

(3) To strengthen the basic information collection of the repaired tubing about service condition, in particular, to clarify whether it has been used in the sulfur well conditions, in order to ensure the safety of the use of repaired tubing.

(4) To enhance the ability of non-destructive testing, improve the accuracy of defect detection, prevent the old pipe with serious defects into the use, so as not to cause oil pipe leakage or cracking.

(5) To develop a new make-break process, reduce or eliminate residual clamp printings on the outer surface of the tubing, so as to improve the surface quality of a pipe body to prevent similar incidents from happening again.

\section{References}

1. S. Wei. Oil-Gas field Surface Engineering 28, 3(2009)

2. Z.C. Li. Pipeline Technique Equipment, 2(2015)

3. GB/T 13298-1991, Metal-Inspection Method of Microstructure

4. GB/T 10561-2005, Steel-Determination of Content of Nonmetallic Inclusions-Micrographic Method Using Standards Diagrams

5. GB/T 6394-2002, Metal-Methods for Estimating the Average Grain Size

6. Y.G. Liu, Q.Y. Luo, S.C. Li, Q.L. Wang. Oil Field Equipment 38,3(2009)

7. D.X. Xia, W. Feng. Natural Gas and Oil 15, 3(1997)

8. H.L. Li, Q.P. Li, Y.R. Feng. Failure Analysis and Prevention of Petroleum Drill Stem(Petroleum Industry Press, Beijing, 1999)

9. M. Li, X.G. Li, H. Chen. Corros. Sci. Prot. Technol. 17, 2(2005)

10. C. Manfredi, J.L. Otegui. Eng. Fail. Anal. 9, 5(2002) 